\title{
NMR Relaxation and ESR Lineshape of Anisotropically Rotating Paramagnetic Molecules
}

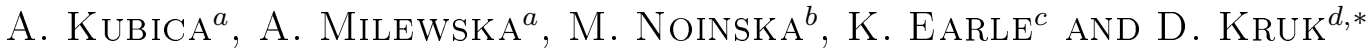 \\ ${ }^{a}$ Institute of Physics, Jagiellonian University, W.S. Reymonta 4, 30-059 Kraków, Poland \\ ${ }^{b}$ The University of Economy in Bydgoszcz, Garbary 2, 82-299 Bydgoszcz, Poland \\ ${ }^{c}$ Physics Department, University at Albany (SUNY), 1400 Washington Ave., Albany, NY 12222, USA \\ ${ }^{d}$ University of Warmia and Mazury in Olsztyn, Faculty of Mathematics and Computer Science \\ Słoneczna 54, PL-10-710 Olsztyn, Poland
}

\begin{abstract}
The role of anisotropic rotation for electron spin resonance lineshape and nuclear magnetic relaxation dispersion profiles for paramagnetic molecules with electron spin quantum number $S \geq 1$ is discussed. The ESR spectra and nuclear magnetic relaxation dispersion profiles are calculated by means of an approach based on the stochastic Liouville equation and referred to in the literature as "Swedish slow motion theory". This description is valid for arbitrary motional conditions and interaction strengths. Molecular tumbling influences the ESR spectra by modulating zero field splitting interactions. The nuclear spin relaxation is affected by the rotational motion in a twofold way: via the electron spin dynamics and as a direct source of modulations of the electron-nuclear dipole-dipole interactions. For coinciding principal axes systems of the permanent (residual, static) zero field splitting and rotational tensors the ESR lineshape is not affected by rotational anisotropy. Rotational anisotropy is important for nuclear relaxation as it is influenced by molecular rotation not only via the electron spin dynamics, but also directly by modulations of the electron spin-nuclear spin dipole-dipole interaction (when the dipole-dipole and zero field splitting frames do not coincide). The anisotropy effects depend strongly on the relative orientation of the dipole-dipole and permanent zero field splitting axes. Nevertheless, a different scenario is also possible. When the diffusion axis coincides with the dipole-dipole axis (but not with the principal axis system of the permanent zero field splitting), the nuclear spin relaxation as well as the ESR lineshape, become sensitive to the rotational anisotropy. The possible dependence of the ESR lineshape and nuclear spin relaxation on the rotational anisotropy should be carefully considered when attempting a joint analysis of ESR and nuclear magnetic relaxation dispersion results for paramagnetic molecules.
\end{abstract}

PACS: 87.64.kj, 87.64.kh, 75.10.Dg, 76.60.Es

\section{Introduction}

Nuclear magnetic resonance (NMR) and electron spin resonance (ESR) are experimental methods well established in molecular science. Careful analysis of resonance spectra can provide detailed information about mechanisms of molecular motion, spin- and molecular interactions provided by appropriate theoretical models are available. For paramagnetic systems (molecules) NMR and ESR serve as complementary techniques. Paramagnetic systems are materials with positive magnetic susceptibility associated with unpaired electrons. Biomolecules containing transition metal ions (for instance paramagnetic metalloproteins) are a good example of such systems. Other examples are transition metal

* corresponding author; e-mail: danuta.kruk@matman.uwm.edu.pl, danuta.kruk@uni-bayreuth.de complexes used as contrast agents in NMR imaging or radicals. Paramagnetic systems of interest for this work contain protons (or other nuclei of spin quantum number $1 / 2$, such as ${ }^{19} \mathrm{~F}$ or ${ }^{13} \mathrm{C}$ ) or are dissolved in proton containing solvents.

In the case of transition metal ions, a hierarchy of interactions is relevant as far as the electron and nuclear spin dynamics is concerned. Due to strong zero field splitting (ZFS) interactions present when the electron spin quantum number $S \geq 1$, the ESR spectra are not affected by the presence of neighboring nuclei. In turn, the electron spin dynamics has a profound effect on the nuclear spin relaxation due to the large magnetic moment of the electron spin (about 650 times larger than the one of protons). A key example is here the effect of paramagnetic relaxation enhancement (PRE) for transition metal complexes (contrast agents) in solution (typically water) $[1,2]$. The PRE effect can be monitored by measuring the ${ }^{1} \mathrm{H}$ spin-lattice relaxation time (rate) of the solvent protons versus magnetic field (frequency). The frequency 
dependence of the spin-lattice relaxation rate is referred to as the nuclear magnetic relaxation dispersion (NMRD) profile $[1,2]$. It can now be routinely measured due to the availability of commercial fast field cycling spectrometers [3] from, e.g., STELAR.

When attempting a quantitative description of the PRE effects including ZFS one faces serious theoretical difficulties. Classical relaxation formulae are derived by applying Redfield relaxation theory [4-6] which is based on second order perturbation theory. This fact causes serious limitations on the interaction strengths and the time scale of the molecular motion that can be studied. The Redfield relaxation theory requires that $\omega^{\prime} \tau_{\mathrm{c}} \ll 1$ [4-6], where $\omega^{\prime}$ is the amplitude (in angular frequency units) of the interaction causing the relaxation while $\tau_{\mathrm{c}}$ is a characteristic time constant (correlation time) describing the time scale of stochastic fluctuations of this interaction. In most cases this condition is rarely fulfilled for the electron spin due to strong ZFS interactions and relatively long rotational correlation times. In fact, the electron spin relaxation can be described by means of perturbation theory only for small molecules in non-viscous solvents. Such systems are of limited interest from the perspective of contemporary applications. Therefore a general theory of the nuclear spin relaxation (PRE), valid beyond the perturbation limit, has been proposed. This treatment is referred in the literature as "Swedish slow motion theory" $[6,7]$. Typically it is used under several simplifying assumptions: the molecular tumbling is treated as isotropic, it is assumed that the ZFS interaction is of axial symmetry and the principal axis of the permanent (static) part of the ZFS tensor coincides with the electron spin-nuclear spin dipole-dipole (DD) axis. It is useful to distinguish a contribution to the ZFS that remains after long-time averaging. Such a contribution is called the permanent ZFS. It is analogous to residual dipole-dipole interactions for anisotropic systems.

The effects of ZFS rhombicity and non-coinciding axes have been discussed [7,9-11]. It has been found that the rhombicity of the permanent ZFS has different effects for integer and half-integer spins due to the Kramers degeneracy for the second case.

Generally, the nuclear spin-lattice relaxation rate (PRE) is reduced in the low field range and this effect is more pronounced for integer spins. However, it also strongly depends on the motional regime (the time scale of the rotational motion). For non-coinciding principal axis systems of the permanent ZFS and the DD axis the nuclear spin-lattice relaxation rate is also reduced in the low field range.

Predictions of the slow motion theory have been compared with other treatments of PRE going beyond the range of validity of the perturbation approach [12]. The result of this comparison confirms the validity of the theoretical treatment discussed here. It has also been extended to include relative translational motion of the electron and nuclear spin (outer-sphere PRE) [13]. Recently, the slow motion theory has been adopted to
ESR lineshape analysis [14] and used for a joint analysis of multifrequency ESR spectra and NMRD for selected $\mathrm{Gd}^{3+}$ complexes [14]. The attempt of a joint, consistent analysis of ESR and NMRD was successful, confirming that the simple model of distortional (vibrational) fluctuations of the ZFS tensor (referred to as pseudorotational model $[1,7,9,10])$ captures essential features of this motion. The molecular tumbling has been treated as isotropic.

The issue whether anisotropy of molecular tumbling can be inferred from ESR lineshapes has been considered for radicals of electron spin quantum number $1 / 2[15,16]$. The NMRD theory for $S \geq 1$ has its origin in the treatment of the ESR lineshape of radicals [16-22], based on the stochastic Liouville equation. In the case of radicals ESR spectra are affected by $g$-tensor anisotropy and scalar interactions of the unpaired electron with neighboring nuclei, for instance ${ }^{14} \mathrm{~N}\left({ }^{15} \mathrm{~N}\right)$ for nitroxyl radicals. The sensitivity of ESR spectra for such systems to the anisotropy of molecular tumbling has been discussed elsewhere $[15,16]$. The effect of anisotropic molecular tumbling on the nuclear spin-lattice relaxation for $S=1$ has been discussed in [11]. It has been pointed out that the rotational anisotropy can influence the relaxation profiles only when the principal axes of the permanent ZFS and DD interactions do not coincide.

In this work we explicitly demonstrate the independence of the ESR lineshape from the rotational anisotropy, in the systems under study, and discuss its influence on nuclear spin relaxation for high electron spin quantum numbers (in analogy to the case of $S=1[11]$ ). It is important to keep in mind the sensitivity of NMRD to the anisotropic tumbling in joint analysis of ESR and NMRD data [14] (even though the ESR spectra remain unaffected). In addition we show examples of ESR spectra for $S=3$ (as an example of integer spin quantum numbers) and show the role of ZFS rhombicity; until now the slow motion theory has been applied to obtain ESR spectra only for $S=7 / 2[14,22]$.

The paper is organized as follows. In Sect. 2 the slow motion theory for NMRD and ESR for $S \geq 1$ is outlined. Its extension to anisotropic tumbling is described. In Sect. 3 the effect of anisotropic rotation on ESR and NMRD is discussed, while Sect. 4 contains concluding remarks.

\section{NMRD and ESR beyond the perturbation limit}

As already explained in Introduction the most important contribution to relaxation processes in paramagnetic systems is due to strong anisotropic ZFS interactions combined with relatively slow molecular tumbling, which prevents a description of the electron spin dynamics by means of well established perturbation theories of relaxation. This implies the necessity of a much more complex approach based on the stochastic Liouville equation. This approach has been described in detail in many papers (for instance $[5,7-14,22]$ ). Here we elaborate on 
only those aspects of the slow motion treatment which are essential for clarity of presentation. Since the electron spin dynamics is independent of the presence of neighboring nuclei we begin with this issue.

In general, there are two types of interactions relevant for the electron spin: the Zeeman coupling (including possible effects of $g$-tensor anisotropy) and ZFS. ZFS interaction can be split into residual (permanent, static) and fluctuating (transient) parts (in fact, these considerations are valid for any spin interaction):

$$
\begin{aligned}
& H_{\mathrm{ZFS}}(t)=\left\langle H_{\mathrm{ZFS}}(t)\right\rangle+\left(H_{\mathrm{ZFS}}(t)-\left\langle H_{\mathrm{ZFS}}(t)\right\rangle\right) \\
& \quad=H_{\mathrm{ZFS}}^{S}+H_{\mathrm{ZFS}}^{T}(t) .
\end{aligned}
$$

The permanent part, $H_{\mathrm{ZFS}}^{S}$, is time independent in a molecule-fixed frame. However, the principal axis system of this interaction $\left(P_{S}\right)$ changes its orientation with respect to the direction of the external magnetic field (the laboratory $(L)$ frame) due to the molecular rotation. The transient part of the ZFS tensor undergoes stochastic fluctuations due to changes in the internal geometry of the molecule which are caused by molecular vibrations, distortions and collisions with surrounding molecules. Figure 1 shows a schematic picture of an electron spin-nuclear spin system including the relevant interactions. $\left(P_{S}\right)$ and $\left(P_{T}\right)$ denote the principal axes systems of the static and transient ZFS, while $\left(D_{R \perp}\right)$ and $\left(D_{R \|}\right)$ are the principal axes of the diffusion tensor. It is assumed that $\left(P_{S}\right)$ and $\left(D_{R \|}\right)$ coincide.

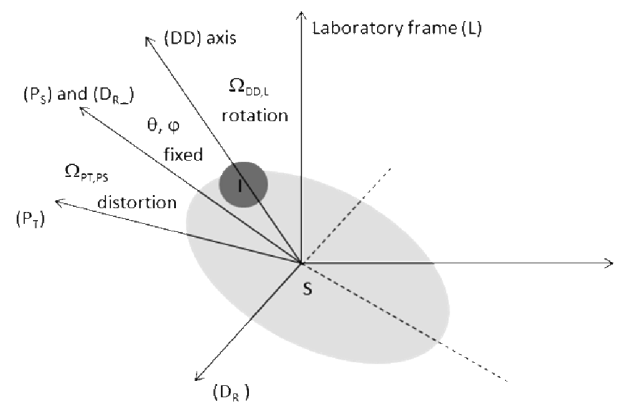

Fig. 1. Schematic view of an electron spin-nuclear spin systems.

This complex physical mechanism has been described by a simple model referred to as the pseudorotational model [5, 7-10, 21]. According to it, the transient ZFS part also has a principal axis system $\left(P_{T}\right)$ which changes its orientation with respect to $P_{S}$ (or any other reference frame fixed in the molecule) following a "pseudorotational" diffusion equation (the free rotation diffusion equation, in fact) with a characteristic time constant, $\tau_{D}$, referred to as the distortional correlation time. This is an obvious simplification which has the advantage of a simple mathematical formulation. Nevertheless, this simple model captures the essential features of the transient ZFS dynamics [14]. The ZFS interactions are characterized by axial and rhombic parameters, $D_{S}, E_{S}$ and $D_{T}, E_{T}$ for the static and transient parts of the tensor, respectively.

The concept of applying the stochastic Liouville equation lies in forming an (in principle) infinite basis set $\left.\left\{\mid O_{i}\right)\right\}$ constructed from the relevant degrees of freedom of the system - in this case distortion $(\mid A B C)$ ), rotation $(\mid L K M))$ and $\operatorname{spin}(\mid \Sigma \sigma))$ variables: $\left.\mid O_{i}\right)=$ $\mid A B C) \otimes \mid L K M) \otimes \mid \Sigma \sigma)[4,6,8,14,22]$.

In this paper we shall not go into the details of the mathematical formulation, they are described elsewhere $[5,7-14,23]$. It is important to note that the ESR lineshape function $L\left(\omega_{S}-\omega\right)$ at the frequency $\omega\left(\omega_{S}\right.$ is the main electron spin (or the Larmor) frequency defined as $\omega_{S}=\gamma_{S} B_{0}$, where $B_{0}$ is the external magnetic field while $\gamma_{S}$ is the electron gyromagnetic ratio) is determined by one element (corresponding to the state $\mid A B C) \mid L K M) \mid \Sigma \sigma)=\mid 000) \mid 000) \mid 1-1)$ ) of an inverted matrix $[M]^{-1}[5,7-10,14,22]$. The matrix is a representation of all the spin interactions (the Zeeman coupling and ZFS, static and transient), as well as the rotational and distortional motions in the basis $\left.\left\{\mid O_{i}\right)\right\}$ obtained by means of the Wigner-Eckart theorem [23]. In the original formulation of the theory the molecular tumbling is treated as isotropic. This implies that the rotational matrix elements of the matrices $[M]$ are given as

$$
\begin{aligned}
& \left(A^{\prime} B^{\prime} C^{\prime}\left|\left(L^{\prime} K^{\prime} M^{\prime}\left|\left(\Sigma^{\prime} \sigma^{\prime}\left|\hat{\hat{L}}_{R}\right| A B C\right)\right| L K M\right)\right| \Sigma \sigma\right) \\
& \quad=\delta_{A A^{\prime}} \delta_{B B^{\prime}} \delta_{C C^{\prime}} \delta_{L L^{\prime}} \delta_{K K^{\prime}} \delta_{M M^{\prime}} \delta_{\Sigma \Sigma^{\prime}} \delta_{\sigma \sigma^{\prime}} \\
& \quad \times \mathrm{i} D_{R} L(L+1)
\end{aligned}
$$

with $\hat{\hat{L}}_{R}=-\mathrm{i} D_{R} \nabla_{\Omega_{P_{S} L}}^{2}$, where $D_{R}$ is the rotational diffusion coefficient related to the rotational correlation time, $\tau_{R}$, via $\tau_{R}=1 /\left(6 D_{R}\right)$. In the present paper we shall consider anisotropic rotational motion for axially symmetric molecules. Then Eq. (2) has to be replaced by

$$
\begin{aligned}
& \left(A^{\prime} B^{\prime} C^{\prime}\left|\left(L^{\prime} K^{\prime} M^{\prime}\left|\left(\Sigma^{\prime} \sigma^{\prime}\left|\hat{\hat{L}}_{R}\right| A B C\right)\right| L K M\right)\right| \Sigma \sigma\right) \\
& \quad=\delta_{A A^{\prime}} \delta_{B B^{\prime}} \delta_{C C^{\prime}} \delta_{L L^{\prime}} \delta_{K K^{\prime}} \delta_{M M^{\prime}} \delta_{\Sigma \Sigma^{\prime}} \delta_{\sigma \sigma^{\prime}} \\
& \quad \times \mathrm{i}\left[D_{R \perp} L(L+1)+\left(D_{R \|}-D_{R \perp}\right) M^{2}\right]
\end{aligned}
$$

where $D_{R \perp}$ and $D_{R \|}$ are the perpendicular and parallel components of the rotational diffusion tensor related to the corresponding rotational correlation times $\tau_{R \perp}=1 /\left(6 D_{R \perp}\right)$ and $\tau_{R \|}=1 /\left(6 D_{R \|}\right)$.

The calculations of the nuclear $\left({ }^{1} \mathrm{H}\right)$ spin relaxation are based on a slightly modified $[M]$ matrix; the frequency $\omega$ is replaced by the proton Larmor frequency $\omega_{l}$. The nuclear spin-electron spin dipole-dipole coupling, which is the origin of the ${ }^{1} \mathrm{H}$ spin relaxation, is modulated, in general, by rotation, the electron spin dynamics and chemical exchange. In the present work we neglect the last effect. The rotational motion plays a double role for the nuclear spin relaxation. It does not only affect the electron spin dynamics but it also directly contributes to the modulations of the dipole-dipole interaction. The form of the DD Hamiltonian determines the elements of $[M]^{-1}$ that describe the nuclear spin-lattice relaxation; 
it is given by a $3 \times 3$ block of the inverted matrix [2, 8-10, $14,22]$. Besides the ZFS parameters and the rotational and distortional correlation times the nuclear spin relaxation depends on the inter-spin distance, $I-S$ (if the exchange processes are neglected), but it plays only the role of a scaling factor. This statement implicitly assumes that the $I-S$ DD axis coincides with the principal axis of the static ZFS. If this is not the case the relative orientation of the two frames has to be introduced into the description of the nuclear spin relaxation [7,9-11]. In the next section we discuss the effects of anisotropic rotation on ESR spectra and ${ }^{1} \mathrm{H}$ NMRD profiles in combination with non-coinciding DD and static ZFS tensors in the context of a unified analysis of both kinds of data.

\section{ESR and NMRD for anisotropic rotation}

The effects of the static ZFS interaction modulated by the rotational dynamics are most pronounced for low-frequency ESR spectra. We begin the discussion with $\mathrm{X}$-band spectra assuming a magnetic field of $0.34 \mathrm{~T}$ with axially symmetric ZFS coupling for $S=3$ (for instance $\mathrm{Mn}^{3+}$ ions). It has generally been assumed that the principal axes systems of the diffusion and static ZFS tensors coincide (as shown in Fig. 1). In Fig. 2a ESR spectra for isotropic rotation with $\tau_{R}=2$ ns have been compared with the case of $\tau_{R \perp}=2 \mathrm{~ns}, \tau_{R \|}=200 \mathrm{ps}$ and $\tau_{R \perp}=200 \mathrm{ps}, \tau_{R \|}=2 \mathrm{~ns}$. One does not see a difference between the first two cases. As explained the ESR spectra are determined by static and transient ZFS interactions which are mediated by the rotational dynamics. (The transformation between the principal axis system of the static ZFS and the laboratory frame arises from the rotational motion. Rotational dynamics also affects the transient ZFS in the transition from the $\left(P_{T}\right)$ frame to the laboratory frame.) However, given that $\left(D_{R \|}\right)$ and $\left(P_{S}\right)$ coincide, rotation around the $\left(D_{R \|}\right)$ axis cannot affect the ZFS interactions, nor, in consequence, the ESR spectra. As expected, for faster molecular tumbling around the perpendicular diffusion axis, $\left(D_{R \perp}\right)$, the structure of the spectrum due to the static ZFS is less pronounced as a result of partial averaging.

Rhombic symmetry of the static ZFS is also of importance for the ESR lineshape. The ESR spectra for the axial and rhombic (assuming the largest possible rhombicity, $E_{S} / D_{S}=1 / 3$ ) cases have been compared in Fig. 2b. Here, too, a faster molecular tumbling around $\left(D_{R \perp}\right)$ reduces the static ZFS structure. The same conclusions are relevant for the case of $S=7 / 2$ (for instance $\mathrm{Gd}^{3+}$ ) shown in Fig. 3a,b. Since the spectra for $\tau_{R}=2 \mathrm{~ns}$ and for $\tau_{R \perp}=2 \mathrm{~ns}, \tau_{R \|}=200 \mathrm{ps}$ are indistinguishable, we do not show in Fig. 3a the first case. It is of some interest to observe the rhombicity effects directly for the absorption spectra as shown in the inset of Fig. 3b.

As explained in Introduction the challenge of combined ESR and NMR studies is a joint analysis of multi frequency ESR spectra and NMRD profiles. Taking into account the double role of the rotational motion as a direct source of modulations of the nuclear spin-electron

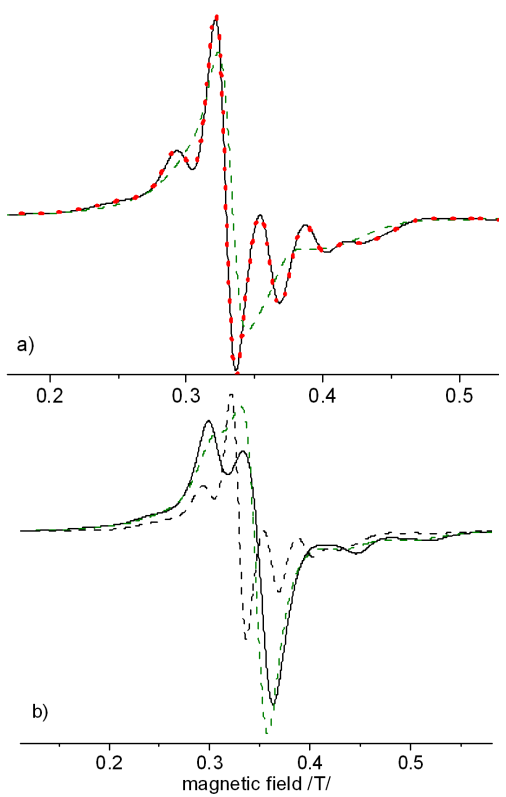

Fig. 2. ESR spectra $(S=7 / 2)$ at $B_{0}=0.34 \mathrm{~T}, D_{S}=$ $0.035 \mathrm{~cm}^{-1}, D_{T}=0.02 \mathrm{~cm}^{-1}, \tau_{D}=20 \mathrm{ps}$; (a) $E_{S}=0$, $\tau_{R}=2 \mathrm{~ns}-$ black solid line; $E_{S}=0, \tau_{R \perp}=2 \mathrm{~ns}$, $\tau_{R \|}=200 \mathrm{ps}-$ red dotted line; $\tau_{R}=200 \mathrm{ps}$ - green dashed line; (b) $E_{S}=D_{S} / 3, \tau_{R}=2 \mathrm{~ns}$ - black solid line; $E_{S}=0, \tau_{R}=2 \mathrm{~ns}$ - black dashed line; $E_{S}=$ $D_{S} / 3, \tau_{R}=200 \mathrm{ps}-$ green dashed line.

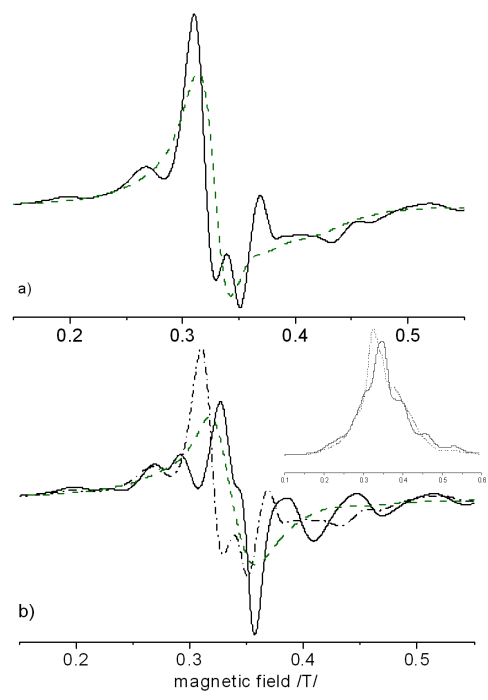

Fig. 3. ESR spectra $(S=7 / 2)$ at $B_{0}=0.34 \mathrm{~T}, D_{S}=$ $0.035 \mathrm{~cm}^{-1}, D_{T}=0.02 \mathrm{~cm}^{-1}, \tau_{D}=20 \mathrm{ps}$; (a) $E_{S}=0$, $\tau_{R}=2 \mathrm{~ns}$ - black solid line; $E_{S}=0, \tau_{R}=200 \mathrm{ps}$ - green dashed line; (b) $E_{S}=D_{S} / 3, \tau_{R}=2 \mathrm{~ns}$ black solid line; $E_{S}=0, \tau_{R}=2 \mathrm{~ns}$ - black dashed line; $E_{S}=D_{S} / 3, \tau_{R}=200 \mathrm{ps}$ - green dashed line. Inset absorption spectra for $E_{S}=D_{S} / 3, \tau_{R}=2 \mathrm{~ns}$ - black solid line and $E_{S}=0, \tau_{R}=2 \mathrm{~ns}$ - black dashed line. 
spin DD coupling and its indirect effect on the DD interaction, one expects that the anisotropic motion considerably influences NMRD profiles through the direct effect. As noted in Introduction this has already been discussed for the case of $S=1$ in [6] pointing out that the rotational anisotropy becomes relevant when the (DD) and $\left(D_{R \|}\right)$ axes do not coincide (angles $\theta, \varphi$ in Fig. 1). In analogy to the ZFS, the dipole-dipole interaction cannot be affected by the motion around an axis coinciding with the DD axis.

In Fig. $4 \mathrm{a}-\mathrm{d}$ the effects of anisotropic rotation on NMRD for different orientations of the (DD) and $\left(D_{R \|}\right)$ axes is shown. If $\tau_{R \|}>\tau_{R \perp}$ the slow molecular tumbling around the parallel axis does not, in any event, lead to significant effects. In the opposite case, $\tau_{R \|}<\tau_{R \perp}$, the faster motion reduces the nuclear spin relaxation for lower magnetic fields to a level depending on the angle $\theta$ between the (DD) and $\left(D_{R \|}\right)$ axes. The chosen angle $\theta=54.74^{\circ}$ is referred to as "magic angle" for the DD interaction, because at this angle the secular (diagonal part) of DD disappears.

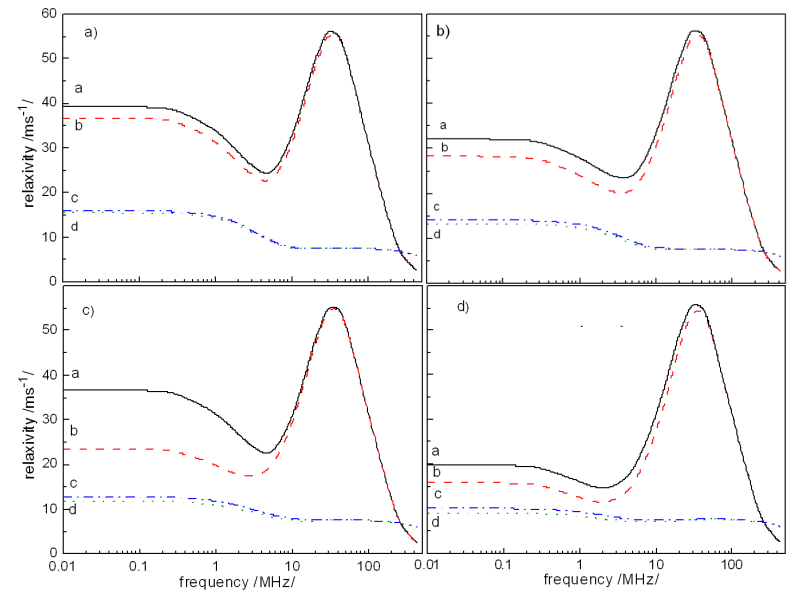

Fig. 4. ${ }^{1} \mathrm{H}$ spin-lattice NMRD profiles for $D_{S}=$ $0.035 \mathrm{~cm}^{-1}, D_{T}=0.02 \mathrm{~cm}^{-1}, \tau_{D}=20 \mathrm{ps}, r_{\mathrm{IS}}=$ $300 \mathrm{pm}$; (a) $\theta=30^{\circ}$, (b) $\theta=45^{\circ}$, (c) $\theta=54.74^{\circ}$, (d) $\theta=90^{\circ}(\varphi=0), \tau_{R}=2 \mathrm{~ns}$ - black line; $\tau_{R \perp}=2 \mathrm{~ns}$, $\tau_{R \|}=200 \mathrm{ps}-$ red dashed line; $\tau_{R \perp}=200 \mathrm{ps}$, $\tau_{R \|}=2 \mathrm{~ns}-$ blue dashed-dotted line, $\tau_{R}=200 \mathrm{ps}-$ green dotted line. Relaxivity is referred to as relaxation for a solvent of $1 \mathrm{~mol}$ concentration.

We wish to stress that the scenario described in this paper where rotational anisotropy does not affect the ESR spectra whereas it can affect NMRD profiles is only one possibility. One can imagine a situation when the (DD) and $\left(D_{R \|}\right)$ axes coincide, whereas $\left(P_{S}\right)$ and $\left(D_{R \|}\right)$ do not (their relative orientation is described by $\theta, \varphi$ ). When this situation obtains, the ZFS interactions become sensitive to the motional anisotropy (they will be affected by rotation around the perpendicular as well as parallel axes because now they will deviate from the principal axes system of the static ZFS) and, in this way, will influence the ESR lineshape. In addition, the nuclear spin relaxation will become affected by the different electron spin dynamics. At the same time the motional anisotropy will not be directly sensed by the DD interaction anymore.

Joint analysis of ESR and NMRD data does not only increase the validity of the dynamical and interaction parameters obtained via such analysis, it also provides support for the motional models used in the analysis. Since ESR spectra and NMRD data can be affected to a different extent by the rotational anisotropy, one should take this account when looking for the best agreement with ESR and NMRD for paramagnetic molecules.

\section{Conclusions}

The influence of anisotropic molecular tumbling on ESR lineshape and NMRD profiles for paramagnetic molecules with electron spin quantum number $S \geq 1$ has been discussed. ESR spectra and NMRD profiles have been calculated by means of "Swedish slow motion theory" based on the stochastic Liouville equation and therefore valid for arbitrary motional conditions and interaction strengths. Examples of ESR spectra for $S=3$ and $S=7 / 2$ for axial and rhombic ZFS are shown. When coincident axes of the static ZFS and rotational diffusion ESR spectra are assumed, it is observed that the ESR spectra are not sensitive to the anisotropy of the molecular tumbling. At the same time NMRD profiles are influenced by this effect (if the DD and diffusion axes do not coincide). Under different circumstances, defined by the relevant stereochemistry, it is possible that both the ESR lineshape and NMRD profiles can become sensitive to the rotational anisotropy. Since ESR and NMR are well established complementary techniques in molecular science, this fact should be kept in mind when evaluating the consistency of motional models applied to joint analysis of ESR lineshapes and NMRD profiles.

\section{Acknowledgments}

This work was supported by funds for science in years 2009-2012 as research project No. NN202 105936 (Polish Ministry of Science and Education).

\section{References}

[1] I. Bertini, C. Luchinat, G. Parigi, Solution NMR of Paramagnetic Molecules, Elsevier, Amsterdam 2001.

[2] J. Kowalewski, D. Kruk, G. Parigi, Adv. Inorg. Chem. 57, 41 (2005).

[3] R. Kimmich, E. Anoardo, Prog. Nucl. Magn. Reson. Spectrosc. 44, 257 (2004).

[4] C.P. Slichter, Principles of Magnetic Resonance, Springer, Berlin 1990.

[5] A.G. Redfield, Adv. Magn. Reson. 1, 1 (1965).

[6] A.G. Redfield, in: Encyclopedia of Nuclear Magnetic Resonance, Ed. D.M. Grant, R.K. Harris, Wiley, Chichester 1996, p. 4085.

[7] T. Nilsson, J. Kowalewski, J. Magn. Reson. 146, 345 (2000). 
[8] N. Benetis, J. Kowalewski, L. Nordenskiöld, H. Wennerström, P.-O. Westlund, Mol. Phys. 48, 329 (1983).

[9] T. Nilsson, G. Parigi, J. Kowalewski, J. Phys. Chem. A 106, 4476 (2002).

[10] T. Nilsson, J. Svoboda, P.-O. Westlund, J. Kowalewski, J. Chem. Phys. 10, 6364 (1998).

[11] N. Benetis, J. Kowalewski, J. Magn. Reson. 65, 13 (1985).

[12] E. Belorizky, P.H. Fries, J. Kowalewski, D. Kruk, R.R. Sharp, P.-O. Westlund, J. Chem. Phys. 128, 052315 (2008).

[13] D. Kruk, J. Kowalewski, J. Chem. Phys. 130, 174104 (2009).

[14] D. Kruk, J. Kowalewski, S. Tipikin, J.H. Freed, J. Moscicki, A. Mielczarek, M. Port, J. Chem. Phys. 134, 024508 (2011).

[15] S.A. Goldman, G.V. Bruno, C.F. Polnaszek, J.H. Freed, J. Chem. Phys. 56, 716 (1972).
[16] J.H. Freed, in: Spin Labeling Theory and Applications, Vol. 53, Ed. L.J. Berliner, Academic Press, New York 1976.

[17] G. Moro, J.H. Freed, J. Chem. Phys. 74, 3757 (1981).

[18] D.J. Schneider, J.H. Freed, Adv. Chem. Phys. 73, 387 (1989).

[19] D.J. Schneider, J.H. Freed, Biol. Magn. Reson. 8, 1 (1989).

[20] Z. Liang, J.H. Freed, J. Phys. Chem. B 103, 6384 (1999).

[21] A. Nevzorov, J.H. Freed, J. Chem. Phys. 112, 1413 (2000).

[22] D. Kruk, Theory of Evolution and Relaxation of Multi-Spin Systems, Arima, Bury St Edmunds 2007.

[23] A.R. Edmunds, Angular Momentum in Quantum Mechanics, Princeton University Press, Princeton 1974. 